\title{
The Effects of Resistance Circle-Ring Exercises with Vibration on the Muscle Thickness with Low Back pain
}

\author{
Jae Cheol Park ${ }^{1}$, Dong Kyu Lee ${ }^{2 *}$ and Seung keun $\mathrm{Oh}^{3}$ \\ ${ }^{1}$ Department of Physical Therapy Chunnam Techno University, Republic of Korea \\ ${ }^{2}$ Department of Physical Therapy Sunhan Hospital, Republic of Korea \\ ${ }^{3}$ Department of Physical Therapy Nambu University, Republic of Korea \\ *Corresponding author: Dong-Kyu Lee, Department of Physical Therapy Sunhan Hospital, Republic of Korea
}

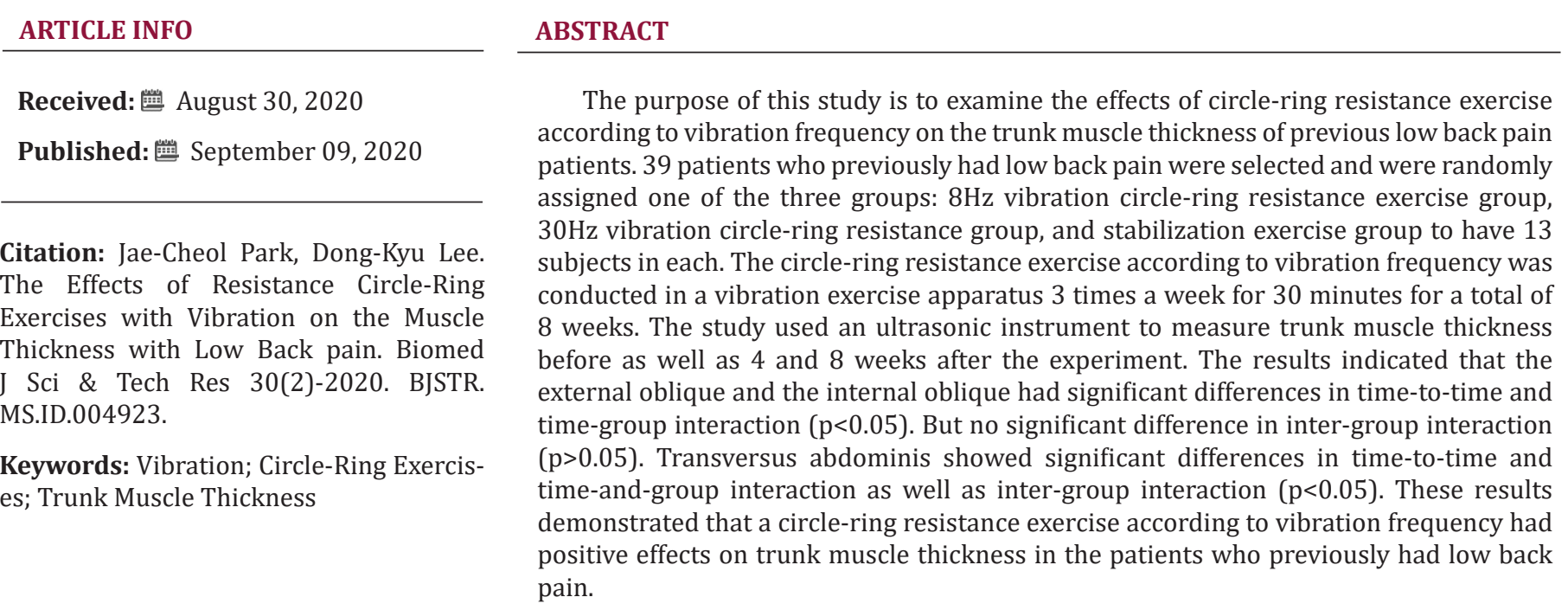

\section{Introduction}

Low back pain is experienced by everyone once in a lifetime and causes significant medical and socioeconomic problems in $20-30 \%$ of the population[1]. People with low back pain generally have decreased range of lumbar motion and show asymmetric movements, expressing difficulties in activities of daily living [2]. However, no neurological causes of low back pain have been precisely identified yet, which makes it difficult to diagnose and treat and leads to a decline in the quality of life beyond pain [3] Numerous variables such as the lack of exercise and anxiety due to pelvic twist, age and stress have drawn attention as the causes of low back pain, and lack of exercise especially causes instability in the lumbar region [4]. The instability of lumbar region weakens muscles and ligament around the spine due to lack of exercise, which thus cannot adequately hold the lumbar region and results in instability, functional disorder, and pain [5].

The muscles related to the lumbar region stability are rectus abdominis and external oblique lateral fiber, which are the surface muscles, and deep muscles are classified into external oblique inner fiber, internal oblique, and transversus abdominis [6]. These muscles are involved in the stability of lumbar spine [4], and the importance of trunk muscles is mentioned to people with lumbar pain; the simultaneous contraction of muscles around the spine is necessary for the stability of spine [7]. Mitra and Mande [8]reported that biofeedback lumbar stabilization exercise for low back pain patients reduces pain during rest and activity. Cruz-Díaz, et al. [9] said that pilates intervention was effective for the exercise anxiety of low back pain patients. Similarly, methods using a stabilization exercise to strengthen the muscles around the lumbar spine have been reported, along with the introduction of exercise method using vibration with easy and huge exercise effects recently.

Vibration is a muscle stimulation method using vibration which has no effects on human body and has recently been proposed for rehabilitation exercise with vibration [10]. Related studies identified that the dual task training applied to stroke patients had 
positive effects on their vastus medialis [11]. They also confirmed the effects of vibration as applying vibration to elderly diabetes patients was related to the improvement of pain level and the flow of leg blood [12]. Studies have also been actively conducted on the effects according to frequency difference, which argued that the frequency difference was reduced by $36.7 \%$ at $20 \mathrm{~Hz}, 28 \%$ at $35 \mathrm{~Hz}$, and $34.8 \%$ at $50 \mathrm{~Hz}$ in the effects on Hoffman reflex according to the frequency difference [13]. Moreover, studies on the effects of vibration exercise on muscular strength with frequency and amplitude difference said that muscular strength was increased in various frequencies [14].

However, there have been insufficient studies on the effects of circle-ring resistance exercise according to a specific vibration frequency on the thickness of trunk muscle for patients with previous low back pain. Thus, this study will present the applicability of resistance exercise using circle-ring as a rehabilitation exercise treatment for patients with previous low back pain, by dividing the vibration frequency into $8 \mathrm{~Hz}$ and $30 \mathrm{~Hz}$ and analyzing the muscles related to low back pain on ultrasonic image.

\section{Materials and Methods}

\section{Subjects Study Design}

This study received Institutional Review Board (No: 1041478 2019-hHR-007) approval from Nambu University's Bioethics Committee and recruited 39 previous low back pain patients through a public announcement to $G$ region in Jeollanam-do Province. The general characteristics of the study subjects were as follows (Table 1). This study explained its purpose and exercise method and constituted its population as people who wished to voluntarily participate in the study and filled out a consent form. The selection of study sample size was calculated by considering significance level ( $\alpha=0.05)$, effect size ( $d=0.8059299)$, and power $(1-\beta=0.95)$ using $G *$ Power $3.1[15]$ based on the results of previous studies [16]. Three groups of 13 people in each of the $8 \mathrm{~Hz}$ vibration circle-ring resistance exercise group (8VCRG), $30 \mathrm{~Hz}$ vibration circle-ring resistance group (30VCRG), and stabilization exercise group (SEG) were formed, calculating 11 in each group and considering the $20 \%$ dropout rate. Information on the groups and exercise effect were not shared with the subjects; the groups were categorized using a simple randomization method by a single-blind test and carried out an exercise in different times for each group. The subjects were selected from those who had experienced lumbar pain within 1 month, those with a VAS score of 2 to 3 , those who do not exercise regularly, and those without any dizziness, vertigo, and problems in the vestibular organs.

Table 1: General characteristics of subject $(n=39)$.

\begin{tabular}{|c|c|c|c|c|}
\hline Characteristics & 8VCRG(n=13) & 30VCRG(n=13) & SEG(n=13) & p \\
\hline Age (year) & $24.46 \pm 3.12$ & $24.61 \pm 3.20$ & $23.61 \pm 2.93$ & 0.209 \\
\hline Height $(\mathrm{cm})$ & $170.08 \pm 8.54$ & $170.31 \pm 8.08$ & $171.46 \pm 7.57$ & 0.141 \\
\hline Weight $(\mathrm{kg})$ & $65.56 \pm 17.96$ & $70.33 \pm 15.20$ & $72.93 \pm 10.64$ & 0.888 \\
\hline
\end{tabular}

8VCRG, $8 \mathrm{~Hz}$ vibration circle-ring resistance exercise group; 30VCRG, $30 \mathrm{~Hz}$ vibration circle-ring resistance exercise group; SEG, stabilization exercise group.

Values are presented as mean \pm standard deviation.

\section{Interventions}

The For $8 \mathrm{~Hz}$ vibration circle-ring (Noble Line Pilates Ring, EVERLAST, TAIWAN) resistance exercise, hip joint flexion, extension, adduction, and abduction exercise were performed at Level $\mathrm{c}$ tension using circle-ring, which is used as a resistance exercise tool (SW-VH16, SONIX, KOREA) in clinical practice on a vibration instrument. The vibration intensity was set at $30 \mathrm{~mm}$, a medium intensity and the frequency was set at $8 \mathrm{~Hz}$. Before starting the exercise, the subjects stretched both feet over the vibration instrument in their shoulder width and flex on the knee joints at $30^{\circ}$ while holding the handles of exercise apparatus to maintain balance. After applying circle-rings between the legs about $10 \mathrm{~cm}$ above the knee joints, a resistance exercise was conducted on the subjects by having them put their legs inside the circle-ring and applying it about $10 \mathrm{~cm}$ above the outer knee joint. After applying the circle-ring about $10 \mathrm{~cm}$ above the knee joint in the straight standing position, the subjects carried out flexion and the extension exercise by flexing and extending each leg alternately.

The exercise program consisted as follows: the participants walked for 5 minutes at a regular walking pace of $3 \mathrm{~km} / \mathrm{h}$ on treadmill as a warm-up, did the main exercise for 1 minute in each exercise, rested for 30 seconds in the middle of each exercise, and stretched for 5 minutes as a cool down. This exercise intervention was applied to the subjects 30 minutes a day, 3 times a week for 8 weeks in total. The suggest that the exercise program of $30 \mathrm{~Hz}$ vibration circle-ring resistance exercise group was the same as the resistance exercise program using $8 \mathrm{~Hz}$ vibration. For stabilization exercise, bridge exercise and plank exercise were applied, with 5 minutes of warm-up, 20 minutes of the main exercise, and 5 minutes of cool down. The main exercise constituted of 30 seconds of isometric exercise and 30 seconds of rest for 10 times.

\section{Experimental Methods Measurements}

This study used an ultrasonic instrument (MyLab25Gold, Esaoth, Italy) to measure muscle thickness. Frequency modulation range was $6 \sim 9 \mathrm{MHz}$, the gain range is $20 \sim 80$, and a $7.5 \mathrm{MHz}$ linear transducer was used for ultrasonic converter. Also, the study measured the muscle thickness after respiration stopped at the final stage of exhalation, taking into account the effects of respiration on the muscle thickness [17]. For the measurement posture, the study subjects lied down straightly to measure their muscle thickness on rectus abdominis, which was $3 \mathrm{~cm}$ away from the navel, and the converter was made horizontally. Next, external oblique, internal oblique, and transversus abdominis were measured by drawing a parallel line along the navel at $45^{\circ}$ flexion of the hip joint and $90^{\circ}$ flexion of the knee joint. The internal oblique was placed in the left edge on the screen of ultrasonic instrument at the point $13 \mathrm{~cm}$ away 
and where the line connecting the anterior superior iliac spine and axillary area met. In order to reduce the error in the measurement, the point was marked with an oil pen and measured by selecting a diagnosis radiologist and one doctor. The average value after a total of three repeated measurements was determined as the muscle thickness.

\section{Statistical Analyses}

Collected data were analyzed using SPSS 19.0 (SPSS Inc., Chicago, IL, USA). Descriptive statistics were used to compare the general characteristics of the participants. All data were verified for normality using the shapiro-wilks verification test. Repeated ANOVA measure was performed to examine changes over time and inter-group differences. As a post analysis, Scheffe's multiple comparison analysis was performed. One-way ANOVA was performed for each inter-group if there was a significant difference in the comparison among groups. The statistical significance level was at 0.05 . In order to find out the difference of each group by time-to-time, the differences values before and after 4 weeks and
8 weeks of experiment were calculated. Also, the significance level was set at 0.025 to reduce type I error.

\section{Results}

The results of repeated measure ANOVA analysis on the external oblique and the internal oblique demonstrated that there was significant difference in time-to-time and time-group interaction $(\mathrm{P}<0.05)$ but no significant difference in inter-group changes $(\mathrm{P}>0.05)$. For the transversus abdominis, there were significant differences in time-to-time and time-and-group interactions and the inter-group interaction $(\mathrm{P}<0.05)$. In terms of time period, the external oblique had a significant difference between 8VCRG and 30VCRG after 4 weeks $(\mathrm{P}<0.05)$. The internal oblique had a significant difference in the 8VCRC after 8 weeks $(\mathrm{P}<0.05)$ and a significant difference in 30VCRG after 4 weeks $(\mathrm{P}<0.05)$. The transversus abdominis had a significant difference in 8VCRG after 8 weeks $(\mathrm{P}<0.05)$ and in 30VCRG after 4 weeks $(\mathrm{P}<0.05)$, and a significant difference in SEG after 8 weeks $(\mathrm{P}<0.05)$ (Tables $2 \& 3$ ).

Table 2: Comparison of trunk muscle thickness.

\begin{tabular}{|c|c|c|c|c|c|c|c|}
\hline & & Pre & 4weeks & 8weeks & Source & $\mathbf{F}$ & $\mathbf{P}$ \\
\hline \multirow{3}{*}{ EO } & 8VCRG & $4.79 \pm 1.66$ & $5.18 \pm 1.48$ & $5.67 \pm 1.31$ & Time & 35.628 & $0.000^{*}$ \\
\hline & 30VCRG & $4.89 \pm 1.14$ & $5.37 \pm 1.29$ & $5.84 \pm 1.20$ & TimeXGroup & 4.775 & $0.002^{*}$ \\
\hline & SEG & $4.68 \pm 1.47$ & $4.76 \pm 1.45$ & $4.86 \pm 1.46$ & Group & 0.682 & 0.512 \\
\hline \multirow{3}{*}{ IO } & 8VCRG & $6.42 \pm 1.55$ & $6.84 \pm 1.61$ & $7.25 \pm 1.29$ & Time & 24.652 & $0.000^{*}$ \\
\hline & 30VCRG & $6.59 \pm 1.65$ & $7.34 \pm 1.73$ & $7.96 \pm 1.68$ & TimeXGroup & 2.511 & $0.049^{*}$ \\
\hline & SEG & $6.48 \pm 1.50$ & $6.67 \pm 1.63$ & $6.86 \pm 1.55$ & Group & 0.607 & 0.55 \\
\hline \multirow{3}{*}{$\operatorname{Tr} A$} & 8VCRG & $2.73 \pm 1.03$ & $2.91 \pm 0.69$ & $3.50 \pm 0.74$ & Time & 60.107 & $0.000^{*}$ \\
\hline & 30VCRG & $2.94 \pm 0.57$ & $3.47 \pm 0.64$ & $4.11 \pm 0.70$ & TimeXGroup & 3.635 & $0.009 *$ \\
\hline & SEG & $2.64 \pm 0.69$ & $2.86 \pm 0.49$ & $3.14 \pm 0.38$ & Group & 3.467 & $0.042 *$ \\
\hline
\end{tabular}

EO, external oblique; IO, internal oblique; TrA, transverse abdominis. 8VCRG, $8 \mathrm{~Hz}$ vibration circle-ring resistance exercise group; 30VCRG, $30 \mathrm{~Hz}$ vibration circle-ring resistance exercise group; SEG, stabilization exercise group.

${ }^{*} \mathrm{P}<0.05$. Values are presented as mean \pm standard deviation.

Table 3: Post-hoc according to interactions of time and groups in 8VCRG and 30VCRG, SEG.

\begin{tabular}{|c|c|c|c|c|c|c|c|}
\hline & & After 4weeks a & $\mathbf{t}$ & $\mathbf{P}$ & After 8weeksb & $\mathbf{t}$ & $\mathbf{P}$ \\
\hline \multirow{3}{*}{ EO } & 8VCRG & $0.39 \pm 0.46$ & 3.081 & $0.010^{*}$ & $0.88 \pm 0.71$ & 4.438 & $0.001 *$ \\
\hline & 30VCRG & $0.47 \pm 0.49$ & 3.456 & $0.005^{*}$ & $0.94 \pm 0.65$ & 5.241 & $0.000^{*}$ \\
\hline & SEG & $0.08 \pm 0.47$ & 0.61 & 0.553 & $0.18 \pm 0.43$ & 1.523 & 0.154 \\
\hline \multirow{3}{*}{ IO } & 8VCRG & $0.41 \pm 1.15$ & 1.289 & 0.222 & $0.82 \pm 0.94$ & 3.153 & $0.008^{*}$ \\
\hline & 30VCRG & $0.75 \pm 0.41$ & 6.496 & $0.000^{* *}$ & $1.37 \pm 0.77$ & 6.361 & $0.000^{*}$ \\
\hline & SEG & $0.22 \pm 0.50$ & 1.589 & 0.138 & $0.41 \pm 0.59$ & 2.521 & 0.027 \\
\hline \multirow{3}{*}{$\operatorname{Tr} \mathrm{A}$} & 8VCRG & $0.17 \pm 0.60$ & 1.047 & 0.316 & $0.76 \pm 0.62$ & 4.396 & $0.001^{*}$ \\
\hline & 30VCRG & $0.52 \pm 0.26$ & 7.15 & $0.000^{* *}$ & $1.16 \pm 0.47$ & 8.725 & $0.000^{* *}$ \\
\hline & SEG & $0.22 \pm 0.50$ & 1.572 & 0.142 & $0.50 \pm 0.45$ & 3.96 & $0.002^{*}$ \\
\hline
\end{tabular}

EO, external oblique; IO, internal oblique; TrA, transverse abdominis. 8VCRG, $8 \mathrm{~Hz}$ vibration circle-ring resistance exercise group; 30VCRG, $30 \mathrm{~Hz}$ vibration circle-ring resistance exercise group; SEG, stabilization exercise group. a Difference between pre and 4weeks. bDifference between pre and 8weeks. ${ }^{*} \mathrm{P}<0.025$.

**P $<0.001$ Values are presented as mean \pm standard deviation 


\section{Discussion}

The purpose of this study is to examine the effects of vibration circle-ring resistance exercise on the trunk muscle thickness of patients with previously low back pain. The size and shape of muscle tissue is different between healthy people and patients with musculoskeletal disorder [18]. Electromyography is used as a method to confirm muscle activity [19], and ultrasound is used to confirm the shape of muscles [20]. Electromyography is more accurate than other equipment in evaluating muscles, but it is difficult and inconvenient to access [21]. On the other hand, ultrasound is used as a testing and evaluation tool because it is highly reliable and accurate for clinical therapists to slice muscle tissues on morphological changes in patients suspected with skeletal diseases [22,23].Results of measuring trunk muscle thickness by ultrasound showed significant differences in time-totime, time-group interaction, and inter-group interaction for the external oblique and internal oblique, while there were significant differences in time-to-time, time-group interaction, and inter-group interaction for transversus abdominis.

The application of a mechanical stimulus such as vibration stimulates the muscle spindle and $\alpha$-execution nerve, causing tonic vibration reflex, which is a reflexive contraction of muscle or tendon, and enhancing the functions of muscles and the nervous system [23]. Abbasi, et al. [24] reported that the trunk muscle strength and endurance increased when vibration was applied to patients with multiple sclerosis, and that the whole-body vibration exercise increased the muscular strength of knee extension. Also, Wirth, et al. [25] reported that a group applying vibration had higher muscle activities in a study comparing the activity of abdominal muscle according to applying vibration. It is difficult to make a direct comparison since there is no study confirming changes in muscle thickness after applying vibration. However, this study showed similar results with preceding studies, which reported that muscle activity change had a high correlation with muscle thickness and muscle activity index can be viewed as muscle thickness index [26].

Vibration is transmitted to the whole body starting from the sole of a foot once it occurs. It is considered that thickness of trunk muscle increases because the contraction of trunk muscle is induced continuously to increase trunk stability, as the vibration destabilizes the support surface. The thickness change of each group by period demonstrated that the group using $30 \mathrm{~Hz}$ of vibration had a significant difference in all muscles after 4 weeks, while the group applying $8 \mathrm{~Hz}$ had a significant difference only in the external oblique from 4 weeks. These results are interpreted into the following several reasons. First, the high frequency at $30 \mathrm{~Hz}$ activates trunk muscles more than the low frequency of $8 \mathrm{~Hz}$. A study of effects on serratus anterior activity in five conditions by dividing the push-up plus exercise, vibration frequency, and amplitude in winging scapula patients reported that the muscle activities of serratus anterior increased at high frequency and high amplitude [27]. Esmaeilzadeh, et al. [28]divided the frequency into $30 \mathrm{~Hz}$ and $50 \mathrm{~Hz}$, measured the dynamic intensity of knee extension, and reported that it increased at $30 \mathrm{~Hz}$ of frequency. At frequencies above $25 \mathrm{~Hz}$, the exercise stimulates more exercise units through the muscle belly, because high vibration is easier to amplify and hence the increase rate of maximum acceleration at high frequency increases as the amplitude increases compared to low frequency $[29,30]$.

Since the increased muscle activity at low frequency results in muscle fatigue and does not increase muscle performance, there is a higher increase of muscle thickness at 30 VCRG than 8 VCRG [31]. Second, various exercise postures showed different exercise effects in each area in addition to frequency and amplitude. Huang, et al. [32] analyzed the transmission force of knees, hip joint, and lumbar spine in three different types of frequencies $20 \mathrm{~Hz}, 30 \mathrm{~Hz}$, and $40 \mathrm{~Hz}$, low and high amplitudes, as well as deep squat posture, high squat posture, and standing posture. He reported that the transmission power to the knees and hip joints decreased as frequency and amplitude increased [32]. Based on these results, it is interpreted that the thickness of trunk muscle was increased in the group which was applied with a high frequency and $30^{\circ}$ flexion of knee joint. Third, a circle-ring resistance exercise applied to thigh activates the trunk muscles. This study conducted a circle-ring resistance exercise for flexion, extension, abduction, and adduction along with vibration. External resistance or movement applied in addition to several exercises further induces the activity of the trunk stabilization muscles [33], while contracting and stabilizing the areas around the spine and minimizing trunk movement [34]. Movements such as the abduction of arm act as trunk agitation, and the arm contracts to the opposite side of the trunk to reduce instability [35]. A leg movement also acts as an external fluctuation, and hip joint abduction adduction causes lateral flexion of the trunk $[34,35]$. Kim, et al. [36]stated that additional resistance to hip joint adduction during a plank exercise increases the muscle activities of external oblique, internal oblique, and transversus abdominis, because the exercise applying circle-ring on the vibrator acts as an external movement and induces the activity of lumbar muscles.

This study investigated the effects of the circle-ring resistance exercise according to vibration frequency on the thickness of trunk muscle in previous low back pain patients and obtained a positive result. However, it is somewhat difficult to make generalizations due to the limited age groups and the previous low back pain patients in a specific region. It is considered necessary to perform continuous, qualitative studies on patients in various age groups and regions as well as on the epidemiological and biochemical changes that were not identified before in the future. It is also hoped that the evidence for positive results identified in this study are applied as a lumbar exercise intervention for previous low back pain patients in clinical settings. 


\section{References}

1. Rice AS, Smith BH, Blyth FM (2016) Pain and the global burden of disease. Pain 157(4): 791-796.

2. Swain CTV, Whyte DG, Ekegren CL, Taylor P, McMaster K, et al. (2018) Multi-segment spine kinematics: Relationship with dance training and low back pain. Gait posture 68: 274-279.

3. Johansson MS, Jensen Stochkendahl M, Hartvigsen J Boyle E, Cassidy JD (2017) Incidence and prognosis of mid-back pain in the general population: A systematic review. Eur J Pain 21: 20-28.

4. Hodges PW, Moseley GL (2003) Pain and motor control of the lumbopelvic region: effect and possible mechanisms. J Electromyogr Kinesiol 13(4): 361-370

5. Larivière C, Shahvarpour A, Gravel C, Gauvin M, Jean AM, et al. (2019) Revisiting the effect of manipulating lumbar stability with load magnitudes and positions: The effect of sex on trunk muscle activation. J Electromyogr Kinesiol 46: 55-62.

6. Miyake Y, Nakamura S, Nakajima M (2014) The effect of trunk coordination exercise on dynamic postural control using a Core Noodle. J bodyw Mov Ther 18(4): 519-525

7. Stevens VK, Bouche KG, Mahieu NN, Coorevits PL, Vanderstraeten GG, et al. (2006) Trunk muscle activity in healthy subjects during bridging stabilization exercises. BMC Musculoskelet Disord 7: 1-8.

8. Mitra M, Mande M (2019) Effectiveness of core stabilization training with pressure biofeedback in the management of mechanical low back pain in subjects between age group of 20-25 years. Indian J Physiother Occup Ther-Int J 13(1): 82-87.

9. Cruz-Díaz D, Romeu M, Velasco-González C (2018) The effectiveness of 12 weeks of Pilates intervention on disability, pain and kinesiophobia in patients with chronic low back pain: a randomized controlled trial. Clin rehabili Trail 32(9): 1249-1257.

10. Rubin C, Judex S, Qin YX (2006) Low-level mechanical signals and their potential as a non-pharmacological intervention for osteoporosis. Age Ageing 35: 32-36.

11. Shih CC, Liao CC, Sun MF, Su YC, Wen CP, et al. (2015) A retrospective cohort study comparing stroke recurrence rate in ischemic stroke patients with and without acupuncture treatment. Medicine 94(39): e1572.

12. Gomes-Neto M, de Sá-Caputo Dd, Paineiras Domingos LL, Marin PJ, Sañudo B, et al. (2019) Effects of Whole-Body Vibration in Older Adult Patients with Type 2 Diabetes Mellitus: A Systematic Review and MetaAnalysis. Can J Diabetes 43(7): 524-529.

13. Laudani L, Mira J, Carlucci F, Orlando G, Menotti F, et al. (2018) Whole body vibration of different frequencies inhibits $\mathrm{H}$-reflex but does not affect voluntary activation. Hum Mov Sci 62: 34-40.

14. Chung P, Liu C, Wang H. Liu Yu, Chuang C, et al. (2017) Various performance-enhancing effects from the same intensity of whole-body vibration training. J Sport Health Sci 6(3): 333-339.

15. Faul F, Erdfelder E, Lang AG, Buchner A (2007) G*Power 3: A flexible statistical power analysis program for the social, behavioral, and biomedical sciences. Behav Res Methods 39(2): 175-191.

16. Mun DJ (2016) The effect of muscle stability exercise on muscle activity, muscle thickness and balance of core muscle. Namwon, Korea, Graduate School of Seonam University.

17. Hodges PW, Gandevia SC (2000) Changes in intra-abdominal pressure during postural and respiratory activation of the human diaphragm. J Appl Physiol 89(3): 967-976.

18. Rahmani N, Mohseni Bandpei MA, Salavati M, Vameghi R, Abdollahi I (2018) Normal values of abdominal muscles thickness in healthy children using ultrasonography. Musculoskelet Sci Pract 34: 54-58.
19. Mohseni Bandpei MA, Nakhaee M, Mousavi ME, Shakourirad A, Reza Safari M, et al. (2014) Application of ultrasound in the assessment of plantar fascia in patients with plantar fasciitis: a systematic review. Ultrasound Med Biol 40(8): 1737-1754.

20. Rahmani N, Mohseni Bandpei MA, Vameghi R, Salavati M, Abdollahi I (2015) Application of ultrasonography in the assessment of skeletal muscles in children with and without neuromuscular disorders: a systematic review. Ultrasound Med Biol 41(9): 2275-2283.

21. Brockmann K, Becker P, Schreiber G, Neubert K, Brunner E, et al. (2007) Sensitivity and specificity of qualitative muscle ultrasound in assessment of suspected neuromuscular disease in childhood. Neuromuscul Disord 17(7): 517-523.

22. Lee JA, Kim SY (2011) Reliability of ultrasonography for the longus colli in asymptomatic subjects. J Kor Phys Ther 23(4): 59-66.

23. Torvinen S, Kannus P, Sievänen H, Järvinen TA, Pasanen M, et al. (2002) Effect of four-month vertical whole body vibration on performance and balance. Med Sci Sports Exerc 34(9): 1523-1528.

24. Abbasi M, Yoosefinejad AK, Poursadeghfard M, Jahromi FP, Motealleh A, et al. (2019) Whole body vibration improves core muscle strength and endurance in ambulant individuals with multiple sclerosis: A randomized clinical trial. Mult Scler Relat Disord 32: 88-93.

25. Wirth B, Zurfluh S, Müller R (2011) Acute effects of whole-body vibration on trunk muscles in young healthy adults. J Electromyogr Kinesiol 21(3): 450-457.

26. McMeeken JM, Beith ID, Newham DJ, Milligan P, Critchley DJ (2004) The relationship between EMG and change in thickness of transversus abdominis. Clin Biomech 19(4): 337-342.

27. Park WY, Koo HM (2018) The effects of vibration frequency and amplitude on serratus anterior muscle activation during knee push-up plus exercise in individuals with scapular winging. J Korean Soc Phys Med 13(4): 67-74

28. Esmaeilzadeh S, Akpinar M, Polat S. Yildiz A, Oral A (2015) The effects of two different frequencies of whole-body vibration on knee extensors strength in healthy young volunteers: A randomized trial. J Musculoskelet Neuronal Interact 15(4): 333-340.

29. Cardinale M, Erskine JA (2008) Vibration training in elite sport: effective training solution or just another fad? Int J Sports Physiol Perform 3(2): 232-239.

30. Choi HH, Lim DH, Hwang SH, Kim YH, Kim HS (2008) A study of biomechanical response in human body during whole-body vibration through musculoskeletal model development. Journal of KSPE 25(5): 155-163.

31. Buss IC, Halfens RJ, Abu-Saad HH (1997) The effectiveness of massage in preventing pressure sores: a literature review. Rehabil Nurs 22(5): 229-242.

32. Huang M, Tang CY, Pang MY (2018) Use of whole-body vibration in individuals with chronic stroke: Transmissibility and signal purity. J Biomech 73: 80-91.

33. Lee DK, Kang MH, Kim JW, Kim YG, Park JH, et al. (2013) Effects of nonparetic arm exercises using a tubing band on abdominal muscle activity in stroke patients. NeuroRehabilitation 33(4): 605-610.

34. Zhang C, Meng X, Anderson DE, Wang W, Tao XY, et al. (2019) Effects of stretch reflex on back muscle response during sinusoidal whole-body vibration in sitting posture: A model study. Int J Ind Ergon 71: 103-110.

35. Mullington CJ, Klungarvuth L, Catley M, McGregor AH, Strutton PH (2009) Trunk muscle responses following unpredictable loading of an abducted arm. Gait Posture 30(2): 181-186.

36. Kim SY, Kang MH, Kim ER, Jung IN, Seo EY, et al. (2016) Comparison of EMG activity on abdominal muscles during plank exercise with unilateral and bilateral additional isometric hip adduction. J Electromyogr Kinesiol 30: 9-14. 


\section{ISSN: 2574-1241}

DOI: $10.26717 /$ BJSTR.2020.30.004923

Dong-Kyu Lee. Biomed J Sci \& Tech Res

(c) (i) This work is licensed under Creative

Submission Link: https://biomedres.us/submit-manuscript.php

$\begin{array}{ll}\text { BIOMEDICAL } & \text { Assets of Publishing with us } \\ \text { RESEARCHES } & \text { Global archiving of articles } \\ \text { - Immediate, unrestricted online access }\end{array}$

